\title{
The Light during Gravitational Super-Compressibility
}

\author{
Kholmurad Khasanov \\ Gas and Wave Dynamics Department, M. V. Lomonosov Moscow State University, Moscow, Russia \\ Email:kholkh@bk.ru
}

Received January 20, 2013; revised February 21, 2013; accepted February 28, 2013

Copyright (C) 2013 Kholmurad Khasanov. This is an open access article distributed under the Creative Commons Attribution License, which permits unrestricted use, distribution, and reproduction in any medium, provided the original work is properly cited.

\begin{abstract}
The interaction of electromagnetic and gravitational fields and gravitational super-compressibility were investigated experimentally. Dynamic emitter provides conditions for the generation of eigenfunctions with eigenvalues for the various fields, including: acoustic, gravitational and electromagnetic. We observe the gravitational waves in gas flowing from the dynamic emitter and their interaction with electromagnetic waves. The gravitational field energy was decreasing when electromagnetic field was emitted through the excitation of condensed medium. The direction of maximum change of the emitted energy of excited medium was strongly opposed to gravity vector at that point. The frequency of radiation against the gravity vector in given point of space exceeded radiation frequency of same source in opposite direction.
\end{abstract}

Keywords: Gravitational Super-Compressibility; Interaction of Electric and Gravitational Fields; Light Frequency Increasing against Gravity Vector

\section{Introduction}

The light of high energy emitted during super-compressibility of supersonic spiraled jets was discussed in [1-4]. High energy field density is detected in gas flowing from the dynamic emitter (nozzle with a central cone). This dynamic emitter is resonator of acoustic, gravitational and electromagnetic waves [3]. The phenomenon of super-compressibility in under-expanded submerged jet on output of the dynamic emitter results to the arising of super-compressed and gravitational waves. These waves structures form the stable boundary layers and emit high energy radiation, as summarized in [5-7].

In our experiments vapor jets form channels of stationary boundary layers with progressing strong compression and condensation of gas due to gravitational super-compressibility. These phenomena are accompanied with emission of high energy radiation from the $\mathrm{X}$-ray to infrared spectrum and in short radio waves regions [8-10].

We detect at first in $[11,12]$ anti-gravitational directed quanta of high energy that emitted from the excited condensed medium against gravity vector in given point.

The interaction of electric and gravitational fields and gravitational super-compressibility were investigated experimentally. In present, paper experimental results are given about shifting emission spectra of the excited gas or condensed medium depending on gravitational field.
Spiraled supersonic jets and incandescent nichrome and wolfram wires and red-hot steel rod in gravitation field were investigated. Separation in space of photons by energy levels were visualized and detected. The phenomenon of gravitational super-compressibility in under-expanded submerged jet on output of the dynamic emitter results to the arising of stationary gravitational waves and is one of case of the separation of light by energy levels.

Alternating super-accelerations arise through the excitation of gas and condensed media. The excited state of the medium and instability of the field generate gravitons and gravitational waves. This field is found in the graviton-photon interaction that provides light emission.

The results of measurement of the changing of energy and frequency of light during gravitational super-compressibility, when light is emitted by the excited gas and condensed medium, are presented in this work. It occurs so that frequency of radiation against the gravity vector in given point of space exceeds radiation frequency of same source in opposite direction.

On the other hand weight of substance is varied at the photon-graviton interaction including when the substance is heating or cooling $[11,12]$.

The distortion of the phase structure of the fields through excitation of the medium leads to the processes of compensating of phase structure changing. 
Phenomena of separation in space of photons by energy levels were visualized and detected.

Alternate super-accelerations of interacting particles in excited medium generate quadruple waves with two polarization directions.

\section{Experimental Part}

\subsection{Separation of Light from the Inclined Supersonic Jet}

Separation of light on the energy levels in gas and condensed media is found, when emission spectra are varying in gravitation field depending on the direction of light emission in reference to vector of gravity. In our experiments the length of the wave structure was equal to 10 diameters of outlet of the nozzle approximately.

The jet with such spiral configuration presents a new type of gravitational waves.

Light emission in fragments of spiral structures of the jet flowing from nozzle with a conic central body was found. In case of the inclined nozzle the light emission with different colors is detected. The bottom and top sides of the jet flowing from the nozzle with central cone are differently glowing (see Figure 1).

\subsection{The Increasing of Light Frequency Emitted against Gravity Vector}

The increasing of the frequency of light in incandescent nichrome and wolfram wires is found. One can see in Figure 2 the increasing of frequency of light on top side of wire. Here gravity vector is perpendicular to wire.

In Figure 2, frequency of radiation from top side directed against the gravity vector exceeds radiation frequency of the wire in the opposite direction. Violet region of glowing is disposed on outside surface, on top line of the wire. Yellow region of glowing is disposed also on outside surface, on bottom line of the wire. And the sides of the wire are glowing with the same frequency.

In Figure 3, there are presented the visualization of incandescent nichrome wire with blue filter and with the same camera. Here top part of glowing space above the wire extends bottom part more than on $60 \%$.

\subsection{Thermal Field of Horizontal Aglow Rod along Gravity Vector}

The distribution of the energy levels of glowing above and below the red-hot steel rod, disposed horizontally was measured. The red-hot steel rod was disposed horizontally on $2 \mathrm{~m}$ from the lab floor. Temperature in different levels under and over the rod was measured (see Figure 4). No convection was detected.

The direct current in the red-hot rod was 100 A, voltage was less than $1 \mathrm{~V}$. Diameter of rod in Figure 4 is 3

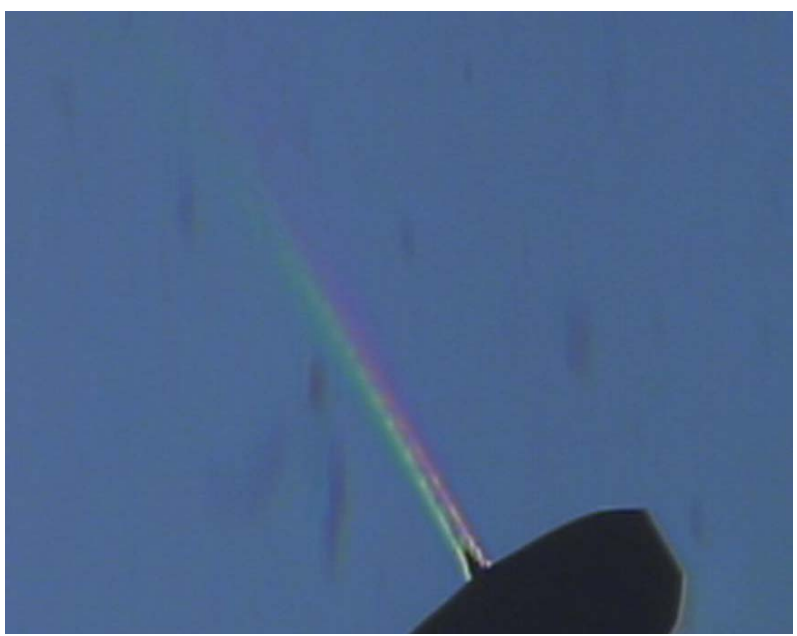

Figure 1. Separation of light emitted from the inclined supersonic jet. The bottom and top sides of the jet flowing from the dynamic emitter of gravitational waves with central cone are differently glowing. Top side of jet is violet with more energy and bottom one is green. In Figure 1, there are no outside electromagnetic sources to excite the jet. The pressure in pre-chamber is 4 atm.
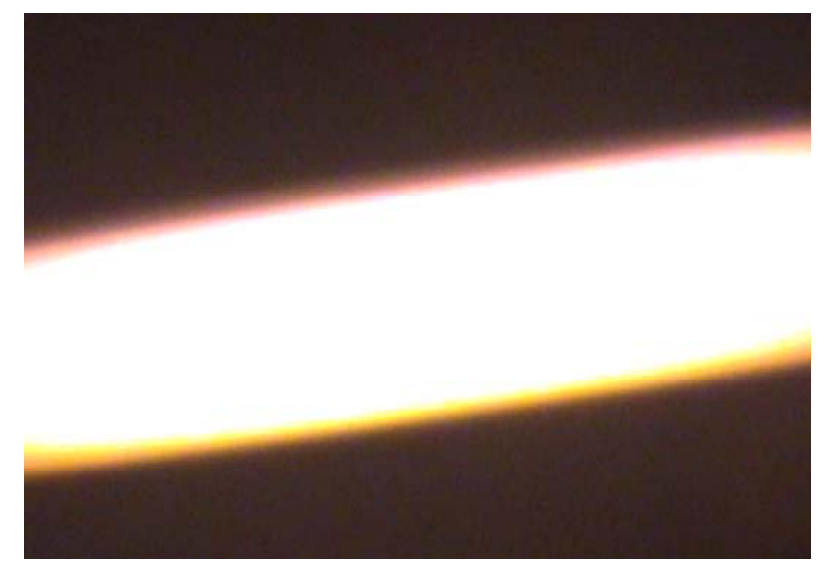

Figure 2. Horizontal spanned incandescent nichrome wire in air has different color on top (violet) and bottom (yellow) sides. Different glowing colors in space are varying along the gravity vector. The diameter of investigated nichrome wire is $1 \mathrm{~mm}$. The length of spanned wire here is $20 \mathrm{~mm}$. Camera SONY Digital Handycam DCR-TRV147E PAL Digital 8 with $560 \times$ digital zoom was used for the visualization.

$\mathrm{mm}$ and its length is $200 \mathrm{~mm}$. Temperature of the air around the rod was measured on the distances from 50 $\mathrm{cm}$ below rod to $60 \mathrm{~cm}$ over the rod. During direct current the temperature remains at same level in given point of space over or under the rod. The charts in Figure 4 describe similar cases with the wire illustrated in Figures $\mathbf{2}$ and $\mathbf{3}$.

Temperature field under and over the rod at the same distances are different. In Figure 4, appropriate temperature for $-50 \mathrm{~cm}$ ( $50 \mathrm{~cm}$ under the rod) is equal $298 \mathrm{~K}$ and 


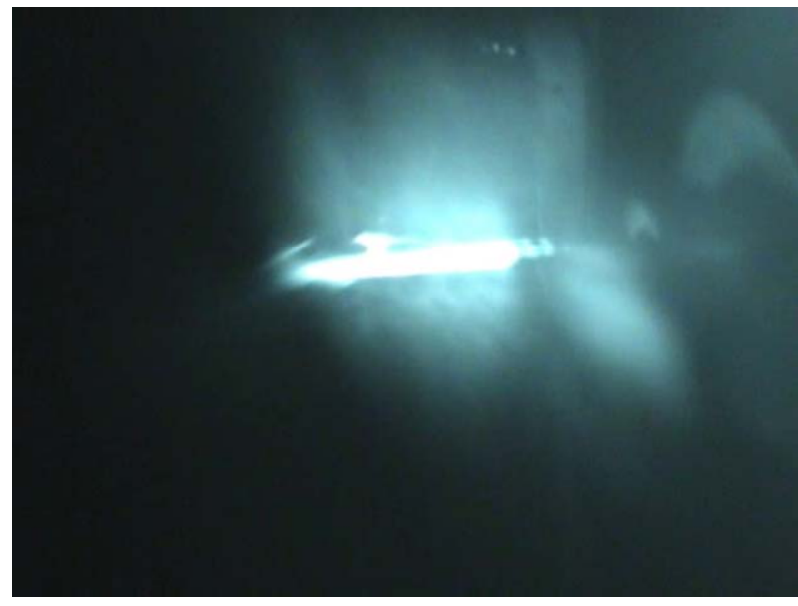

Figure 3. Horizontal spanned incandescent nichrome wire in air with another focusing of camera. The lens of camera is equipped with blue light filter. The diameter of investigated nichrome wire is $1 \mathrm{~mm}$. The length of spanned wire here is $20 \mathrm{~mm}$. Camera SONY Digital Handycam is the same as in Figure 2.

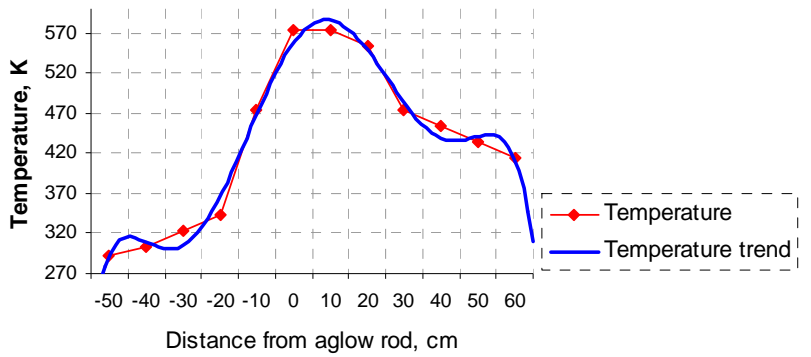

Figure 4. Temperature field distribution above and below red-hot steel rod disposed horizontally.
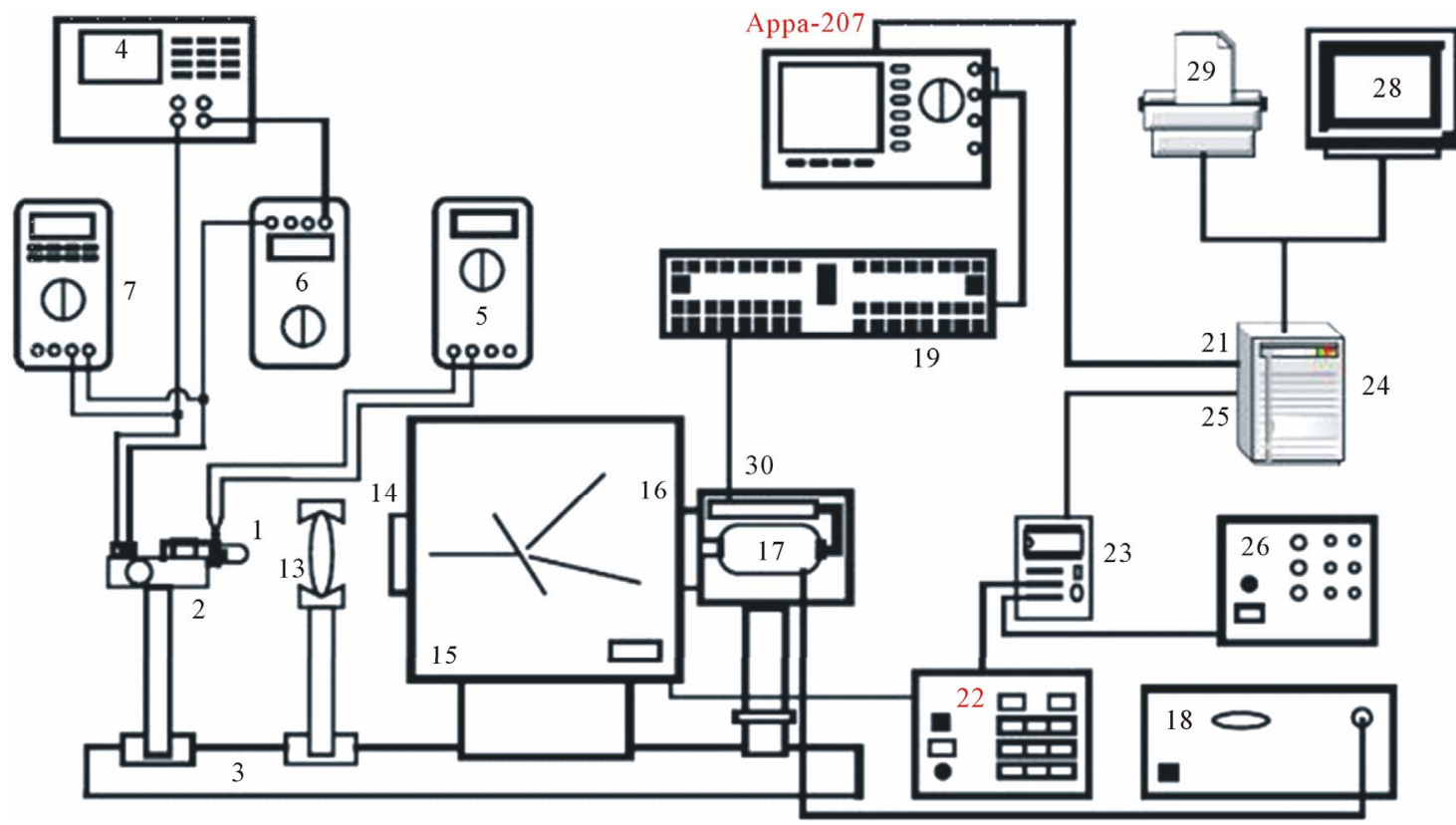

Figure 5. Experimental setup provided spectral measurements and estimate temperature distribution of incandescent wire in lamp in the automatic mode. 
signal intensity at the maximum. Digital power supply Motech LPS-303 allows us to set the current $J$ and voltage $V$ in the lamp within, respectively, from microampere to 2 amperes and from 10 millivolts to 20 volts. $J$ and $V$ were measured with accuracy $0.1 \%$. The voltage across the lamp recorded digital multimeter APPA-101 (7). Multimeter APPA 207 (5) provides detection of temperature on the housing with thermocouples. The radiation from the wire in lamp is focused with collimator (13) on the entrance slit (14) of the diffraction grating monochromator MDR-12 (15). The diameter of the collimating lens is $72 \mathrm{~mm}$ and the distance from the lens to the wire determines the solid angle in which the radiation is focused on the slit.

Determine spectral content of the light passes through the exit slit (16) on the photomultiplier FEU-100 (17), high voltage $(1200 \mathrm{~V})$ of which is supported by the power supply (18). While working on a direct current signal from the photomultiplier goes through a matching circuit (30), goes to the DC amplifier (DCA) (19) and fed to a digital voltmeter ARRA-207 (20) to control the output. The sensitivity of the input stage of DCA ranges from 50 to $1000 \mathrm{nA}$.

Digital signal with a voltmeter goes to the COM port (21) of computer (24) and recorded. Control pulses to the control unit (22) of the monochromator stepper motor come with the interface card. The couplings (23) communicated with a personal computer (24) via a second COM port (25). Voltage of the board (23) implemented via block (26). Experimental setup allows us to investigate the electroluminescence spectra of wire in constant current mode. Then the lamp through the ammeter MA$11 / 5$ (6) to control current value is connected to a universal DC power supply (4), with which you can set the desired voltage through the wire. The voltage across the wire recorded with digital multimeter APPA-101 (7). The figure also shows the following interface devices: the monitor (28) and a printer (29), used in the process of measuring and analyzing the results.

Setup allows the spectra in the automatic mode. The spectra were plotted as chart: "wavelength-intensity" in arbitrary units (see Figures $\mathbf{7}$ and $\mathbf{8}$ ).

In Figures 6 and 7, the emission spectra of horizontal spiral wolfram wire are presented for various voltage and electrical current applied to the wire. Measurement error for wavelength is no more than $0.1 \mathrm{~nm}$. The diameter of investigated spiral wolfram wire in Figures 6-9 is less than $1 \mathrm{~mm}$. The length of wire in lamp is $2 \mathrm{~cm}$.

In Figures 6 and 7, temperature difference $\Delta \mathrm{T}$ between "top" and "bottom" of wolfram wire for same current and voltage is presented. In Figure 8, the uneven along the spectrum character of intensity increasing is obvious. Generation of short-wavelength component $(450 \mathrm{~nm})$ of the spectrum is observed in case of more power of heating.

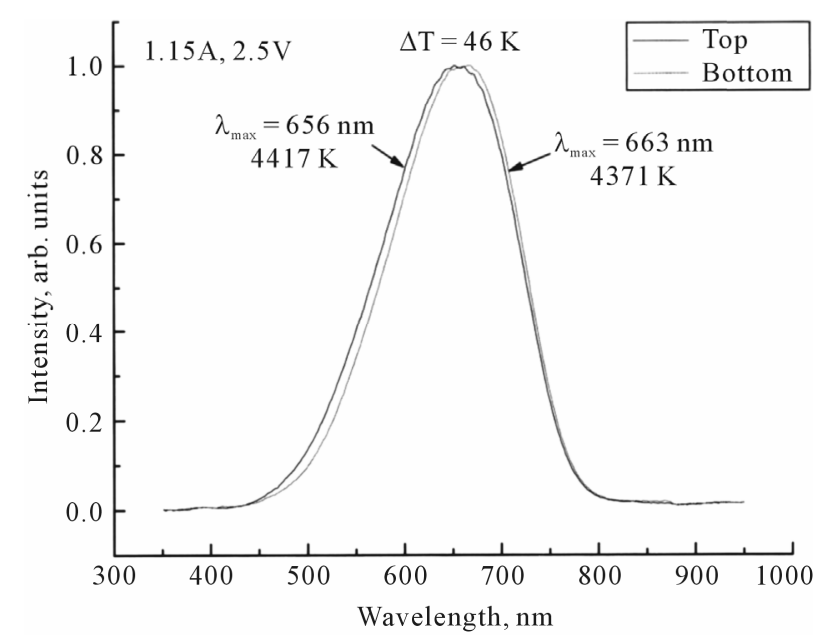

Figure 6. Emission spectra shifting due to frequencies difference from "top" and from "bottom" of the spiral.

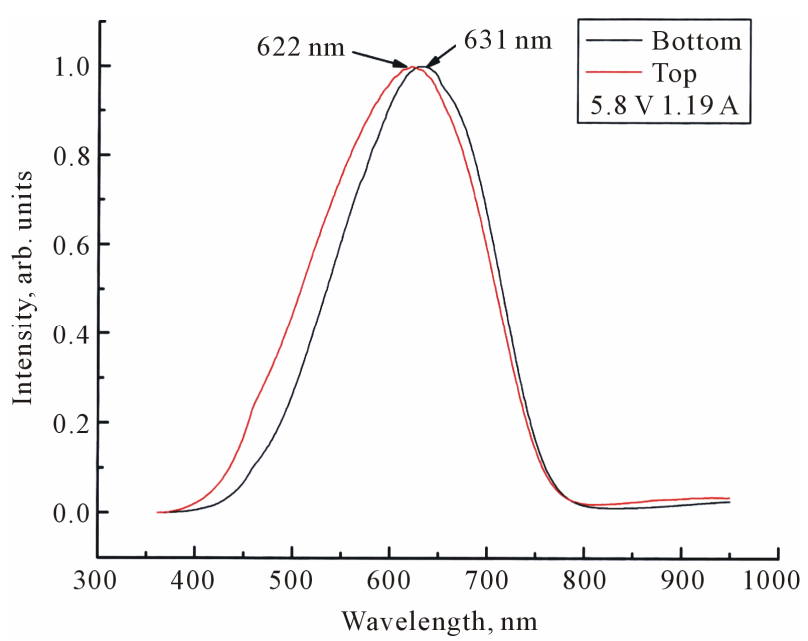

Figure 7. Emission spectra shifting due to frequencies difference from "top" and from "bottom" of wolfram spiral in case of more power of heating.

In Figure 8, the power of heating, resistance and current of spiral wire in lamp versus the voltage on it are presented. Power was received as the product of voltage and current, measured with voltage at the same time.

Measurements show that presented interaction has resonance nature (see Figure 9).

In Figures 8 and 9, there are presented the results of interaction of electromagnetic waves with the background gravitation field.

We observe the gravitational waves in gas flowing from the dynamic emitter and their interaction with electromagnetic waves. The background gravitation field was identified when light was emitted by the excited condensed medium. The direction of maximum change of the eigenvalues of excited medium was strongly opposed to gravity vector at that point. The frequency of radiation against the gravity vector in given point of space exceeds 


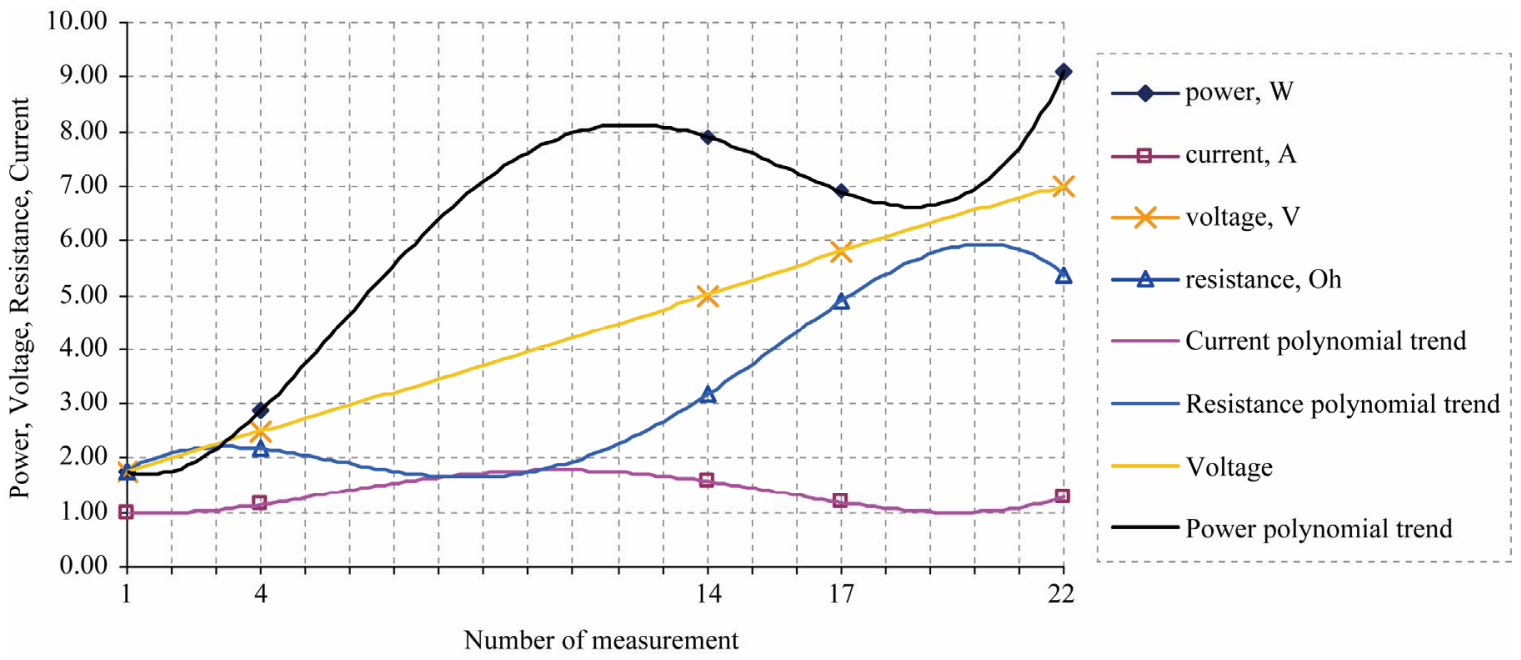

Figure 8. Power of heating, resistance and current of incandescent spiral wolfram wire in lamp versus voltage on it.

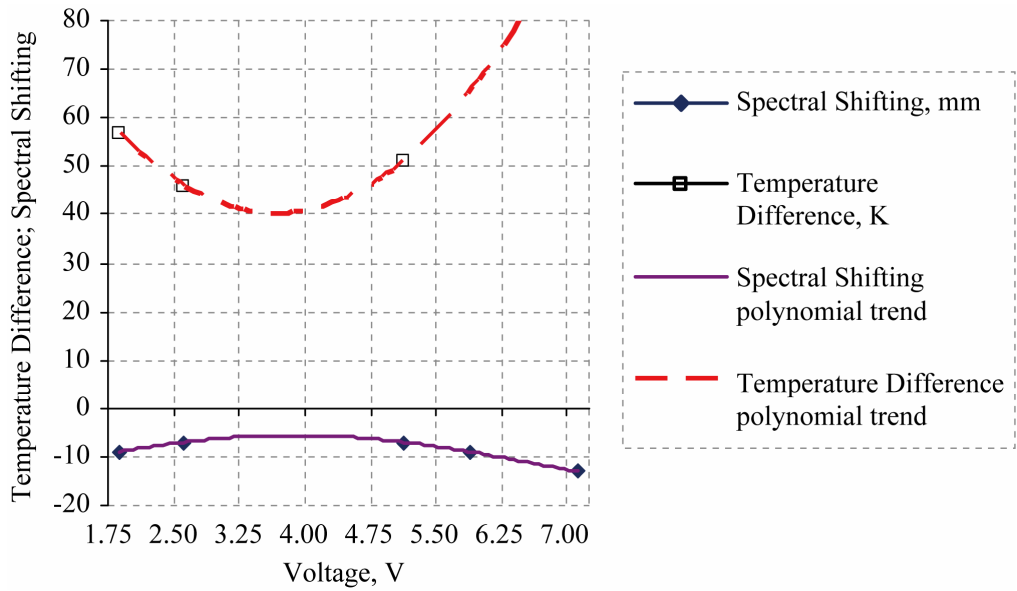

Figure 9. Temperature difference $\left(T_{\text {top }}-T_{\text {bottom }}>0\right)$ between the top and bottom lines of the horizontal wire in the lamp and convenient spectral shifting $\left(\lambda_{\text {top }}-\lambda_{\text {bottom }}<0\right)$ versus voltage on the wire.

radiation frequency of same source in opposite direction. It is result of interaction of background gravitation field with electromagnetic field.

\section{Conclusions}

The interaction of electromagnetic and gravitational fields and gravitational super-compressibility were investigated experimentally.

- Dynamic emitter provides conditions for the generation of eigenfunctions with eigenvalues for the various fields, including: acoustic, gravitational and electromagnetic waves.

- Gravitational super-compressibility of continuous media provides high energy light emission.

- The gravitational field energy was decreasing when electromagnetic field was emitted through the excitation of condensed medium.

- The direction of electromagnetic field gradient was constant with respect to gravity vector at given point of the found alternate background gravitation field.

- The frequency of radiation against the gravity vector in given point exceeds radiation frequency of the same source in the opposite direction.

- Uneven along the spectrum character of intensity increasing is found. Generation of short-wavelength component $(450 \mathrm{~nm})$ of the spectrum is observed in case of more power of heating.

- Measurements show that presented electromagnetic and gravitational interactions have resonance nature.

\section{Acknowledgements}

Author gratefully acknowledges for the long-term support and consulting to V. A. Sadovnichiy, academician of RAS and R. I. Nigmatulin, academician of RAS.

Author deeply appreciates to A. E. Yunovich from M. V. Lomonosov Moscow State University Semiconductor 
Physics Department for kindly provided equipment for spectral measurements.

\section{REFERENCES}

[1] Kh. Khasanov, "Double Spiraled Supersonic Jet," Fluid Dynamics, Vol. 46, No. 3, 2011, pp. 433-436. doi:10.1134/S0015462811030094

[2] Kh. Khasanov, "Bi-Spiral Switched Supersonic Jet Flow Escaping from a Circular Nozzle: Interaction with Metal and Polymer Screens, Infrared Radiation Phenomenon," International Conference: Fluxes and Structures in Fluids. Physics of Geospheres, Moscow, 24-27 June 2009, pp. 206-210.

[3] Kh. Khasanov and S. V. Petukhov, "Dynamic Emitter," PF Patent No. 2058196, 1996.

[4] Kh. Khasanov, "Super-Compressibility Phenomenon," Journal of Modern Physics, Vol. 4, No. 2, 2013, pp. 200-207.

[5] Kh. Khasanov, "Radiation of Bi-Spiral Switched Supersonic Jet Flow Escaping from an Annular Nozzle," In: Fluxes and Structures in Fluids, Physics of Geospheres, Moscow, 24-27 June 2009, 2010, Abstracts.

[6] Kh. Khasanov, "Visualization of Bi-Spiral Twisted Supersonic Gas Flow," The 8th Pacific Symposium of Flow Visualization and Image Processing, Moscow, 21-25 August 2011, p. 51.
[7] Kh. Khasanov, "Visualization of Super-Compressibility in Supersonic Spiral-Twisted Jets," Physics Letters A, Vol. 376, No. 5, 2012, pp. 748-753. doi:10.1016/i.physleta.2011.12.018

[8] Kh. Khasanov, A. I. Klimov, A. S. Pashchina, I. Moralev and M. Sidorenko, "Interaction of Supersonic Jet with Capacity Coupled HF Discharge," The 11th International Workshop on Magneto-Plasma Aerodynamics, Moscow, 10-12 April 2012, pp. 95-96.

[9] Kh. Khasanov, "High-Energy Radiation of Continuous Medium as It Interacts with Sub-Millimeter Fields of Outer Space," The 11th International Workshop on Magneto-Plasma Aerodynamics, Moscow, 10-12 April 2012, pp. 96-97.

[10] Kh. Khasanov, "Emission of High Energy during SuperCompressibility of Supersonic Jets," Open Journal of Fluid Dynamics (OJFD), Vol. 2, 2012, pp. 172-179.

[11] Kh. Khasanov, "Phenomena Taking Place at the Atomic Level under Certain Perturbation," 56th International Symposium on Molecular Spectroscopy, Ohio State University, Columbus, 11-15 June 2001, p. 125.

[12] Kh. Khasanov, "Quantum Antigravitation of Vapor-Air Jet," 56th Meeting of International Symposium on Molecular Spectroscopy, Ohio State University, Columbus, 11-15 June 2001, p. 199. 